\title{
DHSpred: support-vector-machine-based human DNase I hypersensitive sites prediction using the optimal features selected by random forest
}

\author{
Balachandran Manavalan ${ }^{1}$, Tae Hwan Shin ${ }^{1,2}$ and Gwang Lee ${ }^{1,2}$ \\ ${ }^{1}$ Department of Physiology, Ajou University School of Medicine, Suwon, Republic of Korea \\ ${ }^{2}$ Institute of Molecular Science and Technology, Ajou University, Suwon, Republic of Korea \\ Correspondence to: Balachandran Manavalan, email: bala@ajou.ac.kr \\ Gwang Lee, email: glee@ajou.ac.kr
}

Keywords: DNase I hypersensitive site; feature selection; machine learning; random forest; support vector machine Received: September 06, $2017 \quad$ Accepted: November 17, $2017 \quad$ Published: December 08,2017

Copyright: Manavalan et al. This is an open-access article distributed under the terms of the Creative Commons Attribution License 3.0 (CC BY 3.0), which permits unrestricted use, distribution, and reproduction in any medium, provided the original author and source are credited.

\section{ABSTRACT}

DNase I hypersensitive sites (DHSs) are genomic regions that provide important information regarding the presence of transcriptional regulatory elements and the state of chromatin. Therefore, identifying DHSs in uncharacterized DNA sequences is crucial for understanding their biological functions and mechanisms. Although many experimental methods have been proposed to identify DHSs, they have proven to be expensive for genome-wide application. Therefore, it is necessary to develop computational methods for DHS prediction. In this study, we proposed a support vector machine (SVM)-based method for predicting DHSs, called DHSpred (DNase I Hypersensitive Site predictor in human DNA sequences), which was trained with 174 optimal features. The optimal combination of features was identified from a large set that included nucleotide composition and di- and trinucleotide physicochemical properties, using a random forest algorithm. DHSpred achieved a Matthews correlation coefficient and accuracy of 0.660 and 0.871 , respectively, which were $3 \%$ higher than those of control SVM predictors trained with non-optimized features, indicating the efficiency of the feature selection method. Furthermore, the performance of DHSpred was superior to that of state-of-the-art predictors. An online prediction server has been developed to assist the scientific community, and is freely available at: http:// www.thegleelab.org/DHSpred.html.

\section{INTRODUCTION}

Eukaryotic transcription is not only regulated by interactions between transcriptional regulators and cisregulatory DNA elements, but also by chromatin structure, which can affect these interactions. The fundamental unit of chromatins is a nucleosome, which affects transcription; the packing of DNA into nucleosomes inhibits DNA availability to transcriptional regulators [1]. Nucleosomefree regions enhanced with chromatin accessibility, known as DNase I hypersensitive sites (DHSs), have been found predominantly in gene regulatory regions, including promoters, enhancers, and local control regions [2-5]. Since their discovery in 1980, DHSs have been used as markers of regulatory DNA regions $[5,6]$. Therefore, mapping of DHSs has become an effective approach for discovering functional DNA elements in noncoding sequences.

DHSs can be identified through southern blotting technique and chromatin immunoprecipitation, followed by microarray hybridization (ChIP-chip) [7, 8]. However, obtaining information on DHSs using the standard southern blot approach is challenging, time-consuming, and error-prone task, and ChIP-chip is expensive and often time-consuming for genome-wide application. Therefore, it is necessary to develop a novel computational method for identifying DHSs. To this end, Noble et al. proposed a method based on support vector machine (SVM), using nucleotide composition as a feature vector [9]. Feng et $a l$. and Kabir et al. also proposed SVM-based predictors, 
using pseudo-dinucleotide and pseudo-trinucleotide compositions, respectively $[10,11]$. All these methods use a similar approach and none of them is available as a web server or stand-alone tool; their practical value is therefore limited, particularly for experimental biologists. Liu et al. proposed a predictor called iDHS-EL (identifying DHSs by fusing three different modes of pseudo-nucleotide composition into an ensemble learning framework), which combined three independent random forest (RF)based predictors, where each one uses different modes of pseudo-nucleotide composition as input features [12]. iDHS-EL is the only publicly available method different from other approaches.

Although these bioinformatics tools showed encouraging results and stimulated research in this area, further studies are needed for the following reasons. (i) The feature space used by the existing methods to construct models is incomplete and not comprehensive. Hence, other potentially useful features remain to be characterized. (ii) Biologically significant features are intrinsically heterogeneous and multi-dimensional; however, the existing methods do not employ feature selection techniques to quantify the importance and contribution of features used for the model, leading to only a partial understanding of the sequence-DHS relationships. Due to these deficiencies, other methods are needed to accurately predict DHSs in uncharacterized DNA sequences.
In this study, we developed an SVM-based prediction method for DHSs, called DHSpred (DNase I Hypersensitive Site predictor in human DNA sequences), in which the optimal features are selected using RF (see Figure 1 for an overview of the methodology). The optimal feature candidates are selected using RF from a large set of features, which include $k$-mer (mononucleotide composition [MNC], dinucleotide composition [DNC], trinucleotide composition [TNC], tetranucleotide composition [TeNC], and pentanucleotide composition $[\mathrm{PNC}]$ ), dinucleotide physicochemical properties (DPCP), and trinucleotide physicochemical properties (TPCP). In addition to DHSpred, we also developed prediction models using three other machine learning (ML)-based methods (RF, extra-tree classifier [ET], and $k$-nearest neighbor $[k-\mathrm{NN}])$. Our results showed that the performance of DHSpred was superior to that of three ML-based models developed in this study and four state-of-the-art predictors.

\section{RESULTS}

\section{Compositional analysis}

To understand the human nucleotide bias in DHSs and non-DHSs, we performed compositional analysis of $k$-mers (MNC, DNC, TNC, TeNC, and PNC) using the benchmarking dataset. MNC analysis revealed that, on average, guanine $(\mathrm{G})$ and cytosine $(\mathrm{C})$ were dominant in

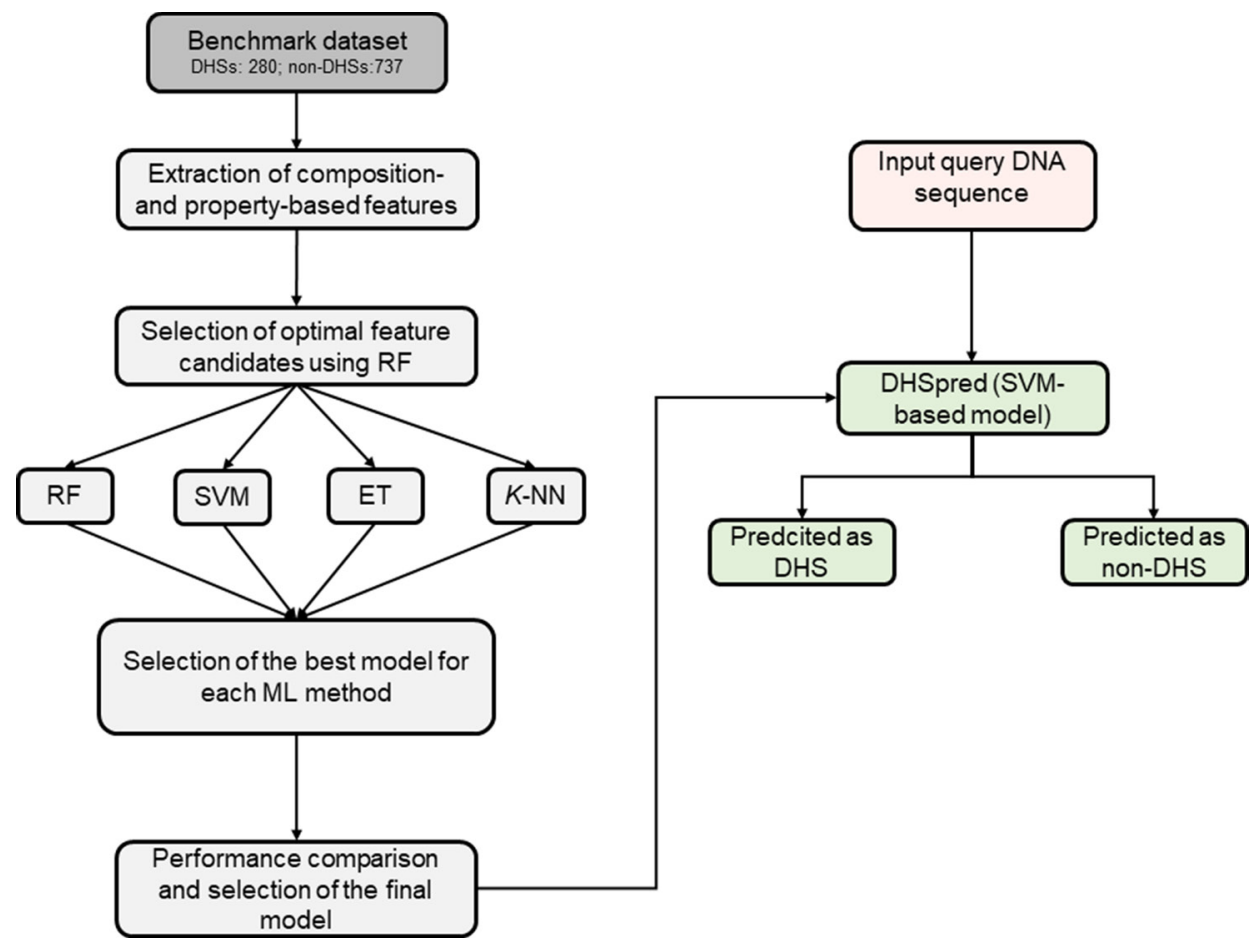

Figure 1: Overall framework of the proposed predictor. Features derived from the DNA sequences, including MNC, DNC, TNC, TeNC, PNC, DPCP, and TPCP, were used as inputs for the RF algorithm to select optimal feature candidates. We then constructed four classifiers based on ML, including RF, SVM, ET, and $k$-NN, using different feature sets, including individual composition, combination of various individual compositions, and feature sets selected from optimal feature candidates. The best model was selected for DHS prediction by comparing the performances of the various models generated in the previous step. 
DHSs, while adenine (A) and thymine (T) were dominant in non-DHSs (Welch's $t$-test; $P \leq 0.01$ ) (Figure 2A). This trend was also reflected in DNC analysis, where 3 of the top 5 motifs (CG, GC, AT, CC, and TG) abundant in DHSs contained these two ( $\mathrm{C}$ and $\mathrm{G}$ ) mononucleotides (Figure 2A), when sorted based on the absolute difference in composition between DHSs and non-DHSs. Furthermore, we observed that $69 \%, 60 \%$, and $40 \%$ of TNC, TeNC, and PNC, respectively, differed significantly between DHSs and non-DHSs (Welch's $t$-test; $P \leq 0.01$ ). Among them, the top five motifs from TNC (CGC, GCG, CCG, CGG, and GCC), TeNC (CGCC, CCGC, GCGC, GCGG, and GGCG), and PNC (CCGCC, CGCCC, CCGGG, CCCGC, and GGCGG) were from DHSs (Figure 2B, 2C, and 2D) and contained different combinations of $\mathrm{C}$ and $\mathrm{G}$. Previous experimental studies have also shown that GC-rich regions showed an open and relaxed chromatin state, which will be convenient for the binding of other macromolecules [1315]. Results from the compositional analyses suggested that integrating the amino acid preference information would be helpful for differentiating between DHSs and non-DHSs, and so, we used these as input features for ML methods to improve classification. The major advantage of ML methods is their ability to consider multiple features simultaneously, often capturing hidden relationships [16-23].
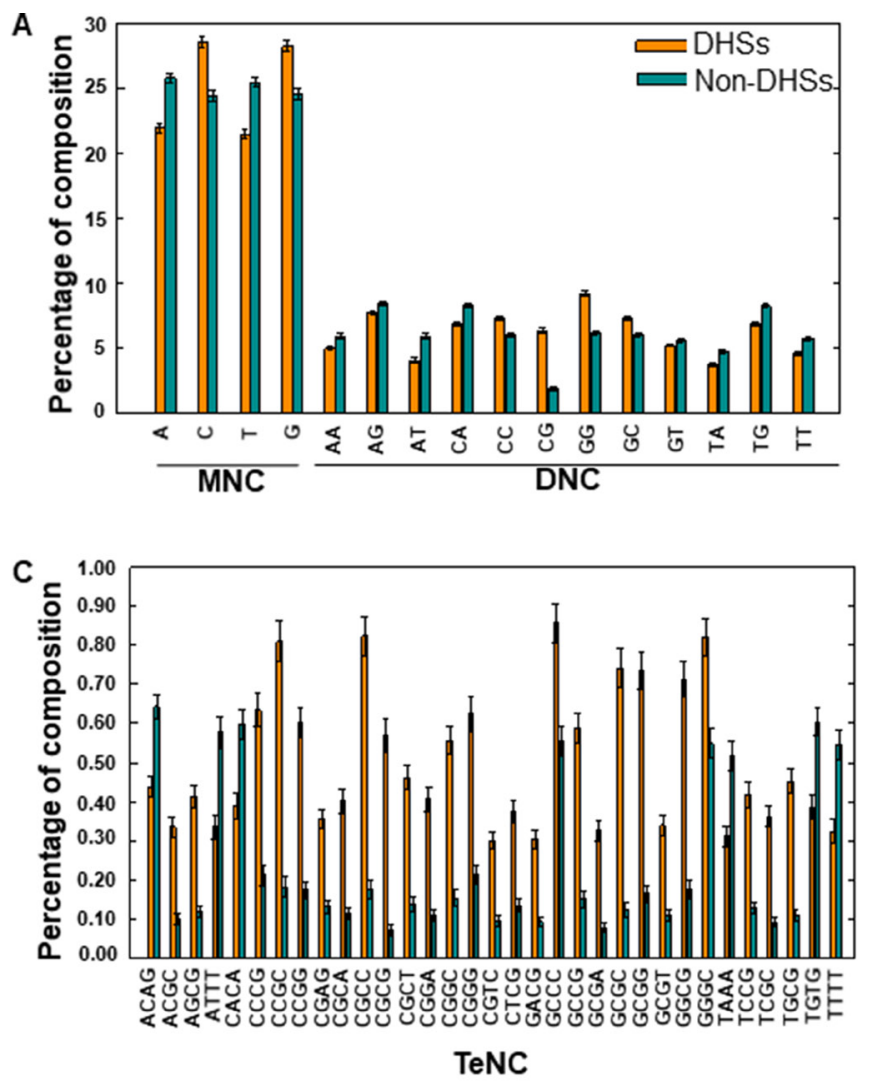

\section{Construction of an SVM-based model using the optimal feature set}

An SVM-based model was constructed using the optimal feature set, through two steps. In the first step, we employed the RF algorithm and evaluated the importance of 2228 features (MNC: 4; DNC: 16; TNC: 64; TeNC: 256; PNC: 1024; DPCP: 94; and TPCP: 768) for distinguishing DHSs from non-DHSs. Based on a threshold feature importance score (FIS) $\geq 0.0003,1139$ features were selected as optimal feature candidates (Figure 3A and Supplementary Table 1). The percentage of individual contributions to the optimal feature candidates is shown in Figure 3B; most of the contribution was from TPCP, PNC, and TeNC. In the second step, to select more important features, we generated 19 sets of features from the optimal feature candidates using an FIS cut-off $(0.0003$ $\leq$ FIS $\leq 0.0021$, with a step size of 0.0001$)$. SVM-based prediction models corresponding to these features were then developed. The performance of these 19 prediction models, in terms of Matthews correlation coefficient (MCC), is shown in Figure 3C, where the performance peaked with 174 features (F174). Therefore, we considered this model final, with the optimal feature set. Furthermore, we examined the percentage of individual contributions in the optimal feature set. As shown in Figure 3D, TPCP
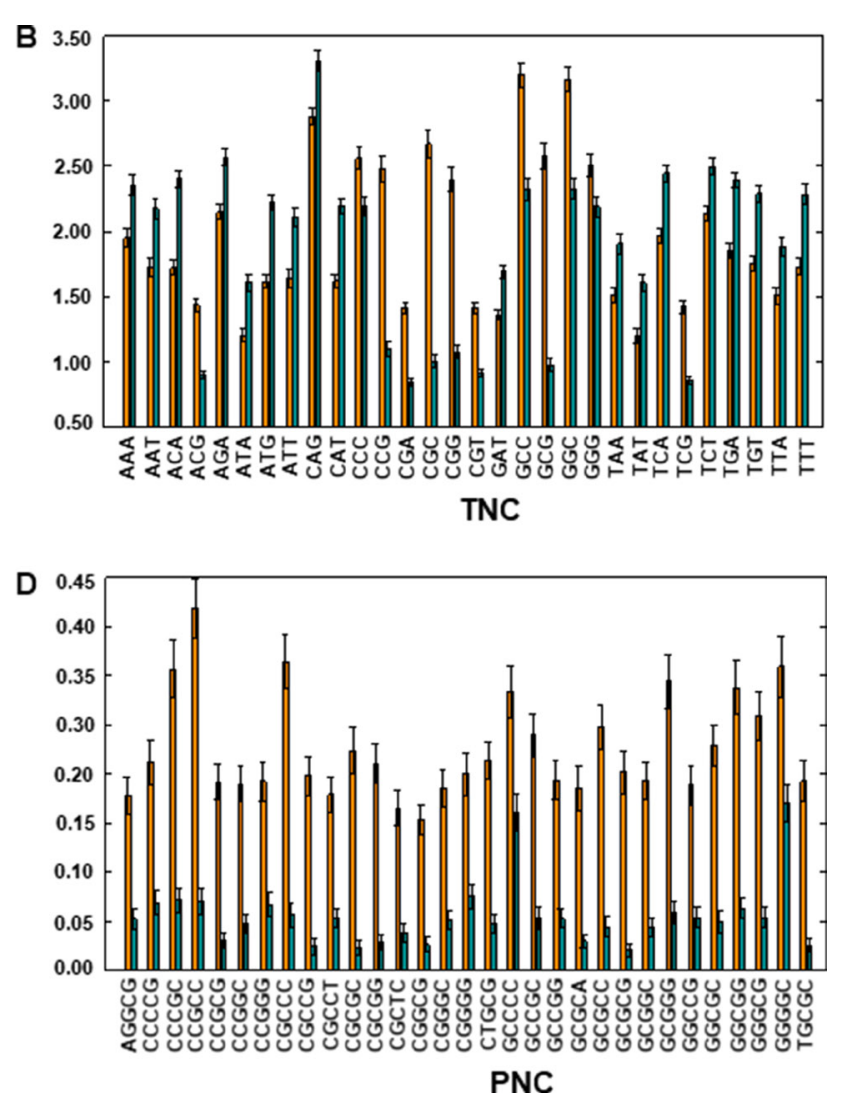

Figure 2: Compositional analysis. (A) MNC and DNC preferences in DHSs and non-DHSs. TNC, TeNC, and PNC preferences between DHSs and non-DHSs is shown in (B), (C), and (D), respectively. In (B), (C), and (D), only compositions with absolute differences between DHSs and non-DHSs of greater than 0.20 are shown. 
introduced in this study contributed $51.4 \%$, followed by PNC (20\%), TeNC (4\%), DPCP (10\%), TNC (12.6\%), and DNC $(2 \%)$, indicating that TPCP played a major role in distinguishing DHSs from non-DHSs.

Generally, it might be possible for hybrid models (combination of individual compositions) or individual composition-based models to perform better than models developed by rigorous feature selection protocols, such as the one described above. To investigate this possibility, we developed prediction models using individual composition or hybrid models, whose performances in terms of MCC, accuracy, sensitivity, and specificity are shown in Figure 4A. The results showed that the F174-based model was superior to the other models; the F174 model had an MCC value of 0.660 , which was $3 \%$ and $2-4 \%$ higher than that of the control SVM predictors (non-optimized features or all features) and individual composition-based or hybrid models, respectively. In addition, the number of selected features decreased from 2228 to 174 . These results indicated that our feature selection protocol was effective for identifying important and informative features. By removing redundant and less informative features through FIS-based feature selection, we could effectively improve the performance of our model.

\section{Comparison of three ML-based models with the SVM-based model}

In the second step of the previous section, we used three different ML-based methods instead of SVM, including, RF, ET, and $k$-NN. A detailed description of the development of prediction models using these methods was provided in our recent studies [22]. For each ML-based method, we generated 33 prediction models using different sets of features, including individual composition, hybrid models, and features based on FIS cut-off. The detailed performance of these methods with respect to different feature sets is shown in Figure 4B-4D). Subsequently, we compared the performance of the three ML-based models with that of the SVM-based model. Interestingly, we observed that the overall performance of the SVM-based model was superior to that of the three ML-based models (ET, RF, and $k-\mathrm{NN}$ ), irrespective of the features used. This result indicated that the SVM method was more suitable for predicting DHSs than other MLs (Figure 5). We then selected the best model from each ML-based method, based on the highest MCC, whose performance is shown in Table 1. Similar to SVM, RF- and $k$-NN-based methods also showed their best performance

A

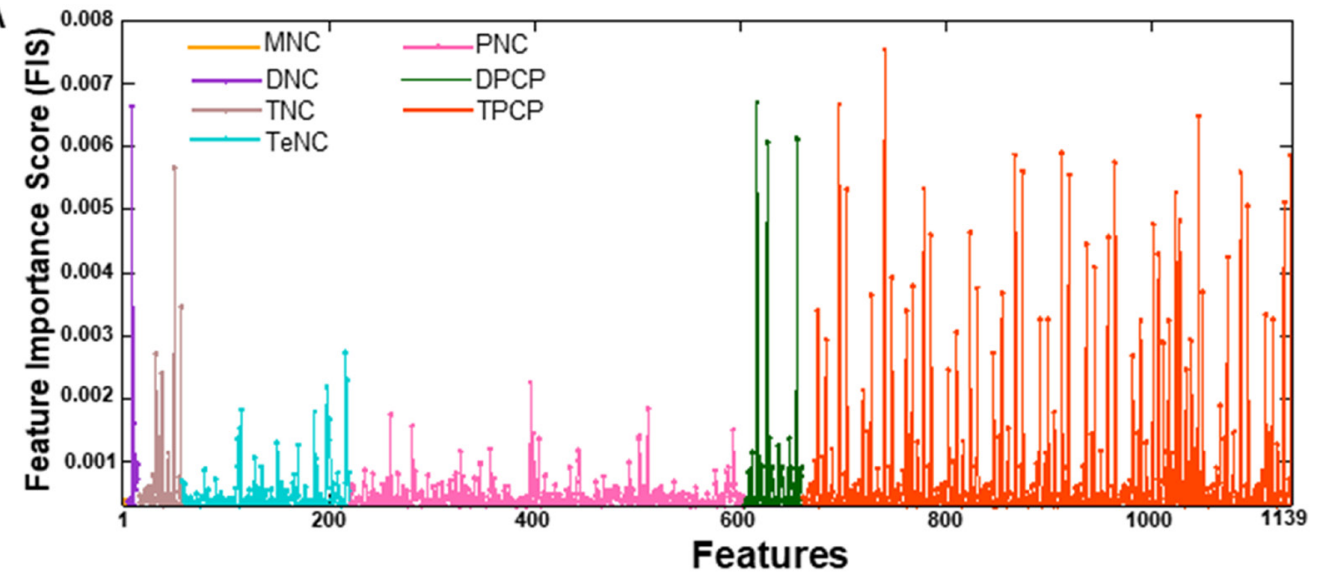

B

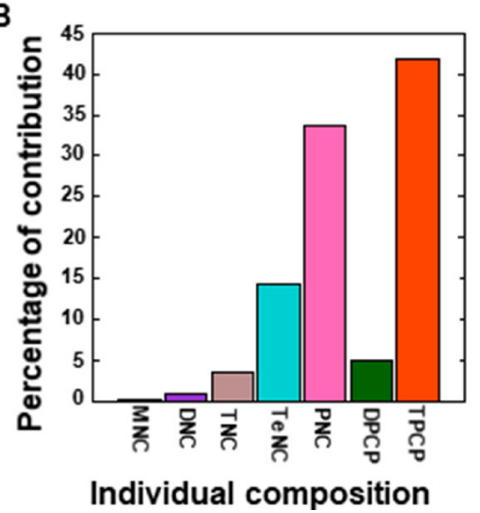

C

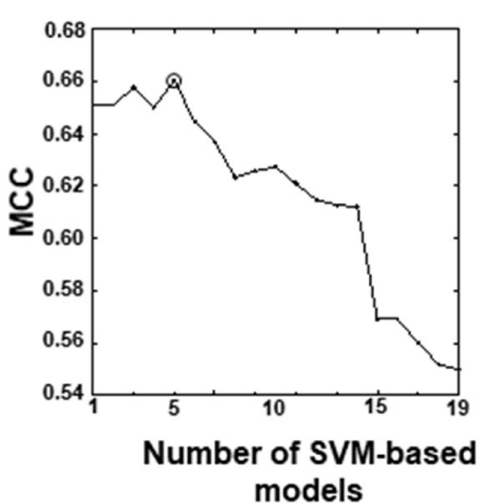

D

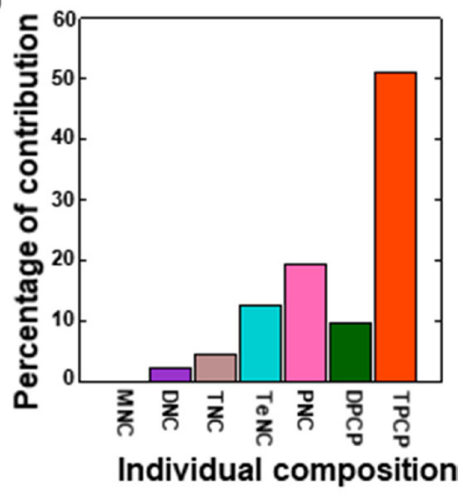

Figure 3: (A) Optimal feature candidates are shown along with their importance scores. The X-and Y-axes represent the features and their importance scores, respectively. The percentage of individual contributions of the optimal feature candidates is shown in (B). (C) The performance of SVM-based models with respect to 19 different sets of features, which were generated from optimal feature candidates using FIS cut-offs. The final selected SVM-based model is circled, and its feature contribution is shown in (D). 
Table 1: Performance of the best selected model from each ML-based method

\begin{tabular}{ccccccc}
\hline Method & MCC & Accuracy & Sensitivity & Specificity & Pt & Py \\
\hline SVM & $\mathbf{0 . 6 6 0}$ & $\mathbf{0 . 8 7 1}$ & 0.655 & $\mathbf{0 . 9 5 2}$ & 0.624 & $\mathbf{0 . 6 0 7}$ \\
RF & 0.638 & 0.861 & $\mathbf{0 . 6 6 9}$ & 0.934 & $\mathbf{0 . 6 2 5}$ & 0.603 \\
ET & 0.635 & 0.848 & 0.668 & 0.931 & 0.622 & 0.599 \\
$k$-NN & 0.632 & 0.860 & 0.642 & 0.943 & 0.605 & 0.585 \\
\hline
\end{tabular}

The first column represents the ML-based method developed in this study. The second, the third, the fourth and the fifth, the sixth and the seventh respectively represent the MCC, accuracy, sensitivity, specificity, Pt and Py. Bold font denotes the best result.

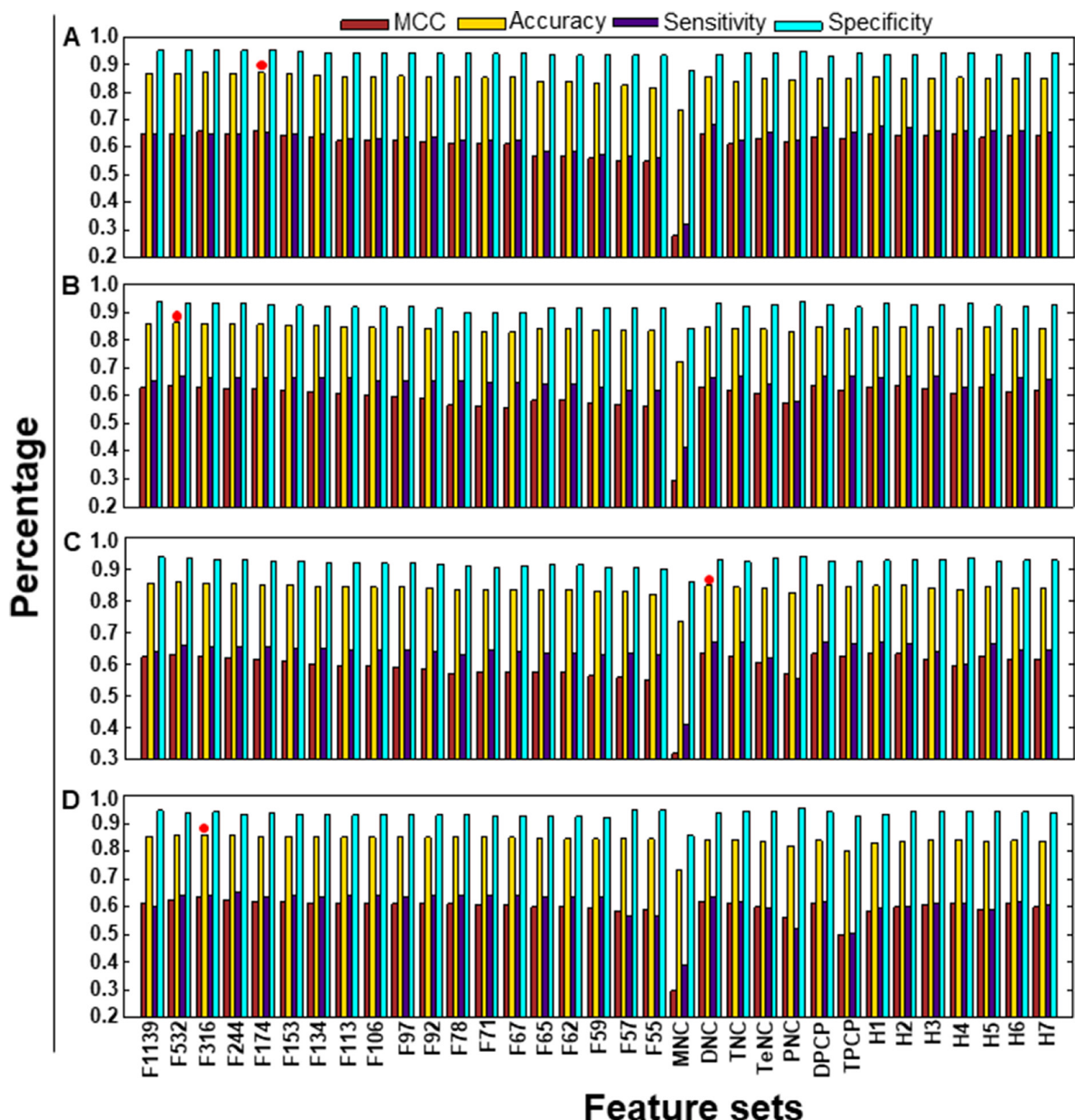

Figure 4: Performance of four different ML-based classifiers. Performance of various classifiers for distinguishing between DHSs and non-DHSs. A total of 33 classifiers were evaluated using five independent 10 -fold cross-validation techniques, and their average performances in terms of MCC, accuracy, sensitivity, and specificity are shown. (A) SVM-based performance, (B) RF-based performance, (C) ET-based performance, and (D) $k$-NN-based performance. The final selected model for each ML method is shown in red filled circle. 
when optimal feature sets were selected using different FIS cut-offs. This observation highlights the importance of our feature selection protocol. However, in terms of MCC and accuracy, the SVM-based model was superior to the other ML-based methods (RF, ET, and $k$-NN) by $\sim 2 \%$ and $1-2 \%$, respectively.

To provide an intuitive comparison among the four methods, we plotted the receiver operating characteristics (ROC) curve of these four methods (Figure 6). Here, the AUC (area under the ROC curve) value higher is the better. Results shows that again SVM-based model showing the superiority over other three ML-based methods (RF, ET, and $k-\mathrm{NN}$ ) in terms of AUC.

\section{Comparison of state-of-the-art predictors with the SVM-based model}

In order to test the quality of the performance of our SVM-based model (DHSpred), it was necessary to compare it to other state-of-the-art methods. Here, we compared it with four such methods (RevcKmer, PseDNC, PseTNC, and iDHS-EL), which were developed from the same benchmark dataset [9-12]. The results are shown in Table 2, in which the methods are ranked according to MCC, which reflects the prediction capability. DHSpred was ranked highest, with MCC, accuracy, sensitivity, specificity, product of sensitivity and specificity $(\mathrm{Pt})$, and product of excess (Py) values of $0.660,0.871,0.655$, $0.952,0.624$, and 0.607 , respectively. For all six metrics (MCC, accuracy, sensitivity, specificity, Pt, and Py), our method had higher scores than the other methods by $2-9 \%$, $1-3 \%, 0.1-4 \%, 1-3 \%, 1.5-6 \%$, and $2-7 \%$, respectively. This result suggested that DHSpred was more effective and robust for identifying DHSs.

The superior efficiency of DHSpred could be attributed to the fact that it selectively incorporates multiple aspects of important information (achieved through systematic feature selection) from $k$-mer, DPCP, and TPCP, while most other methods use all available information. For instance, iDHS-EL considers all the features from DPCP, instead of merely the important motifs. Although the existing methods could achieve relatively high-level performance, further improvement in performance is limited when handling complicated data samples. Hence, incorporating important features from multiple complementary sources of information (as the DHSpred method does) could allow us to sufficiently capture the differences between true DHSs and non-DHSs, thus improving the predictive performance.

\section{DHSpred online prediction server}

As mentioned in previous publications [20, 24-26], a prediction method along with its web server would be of

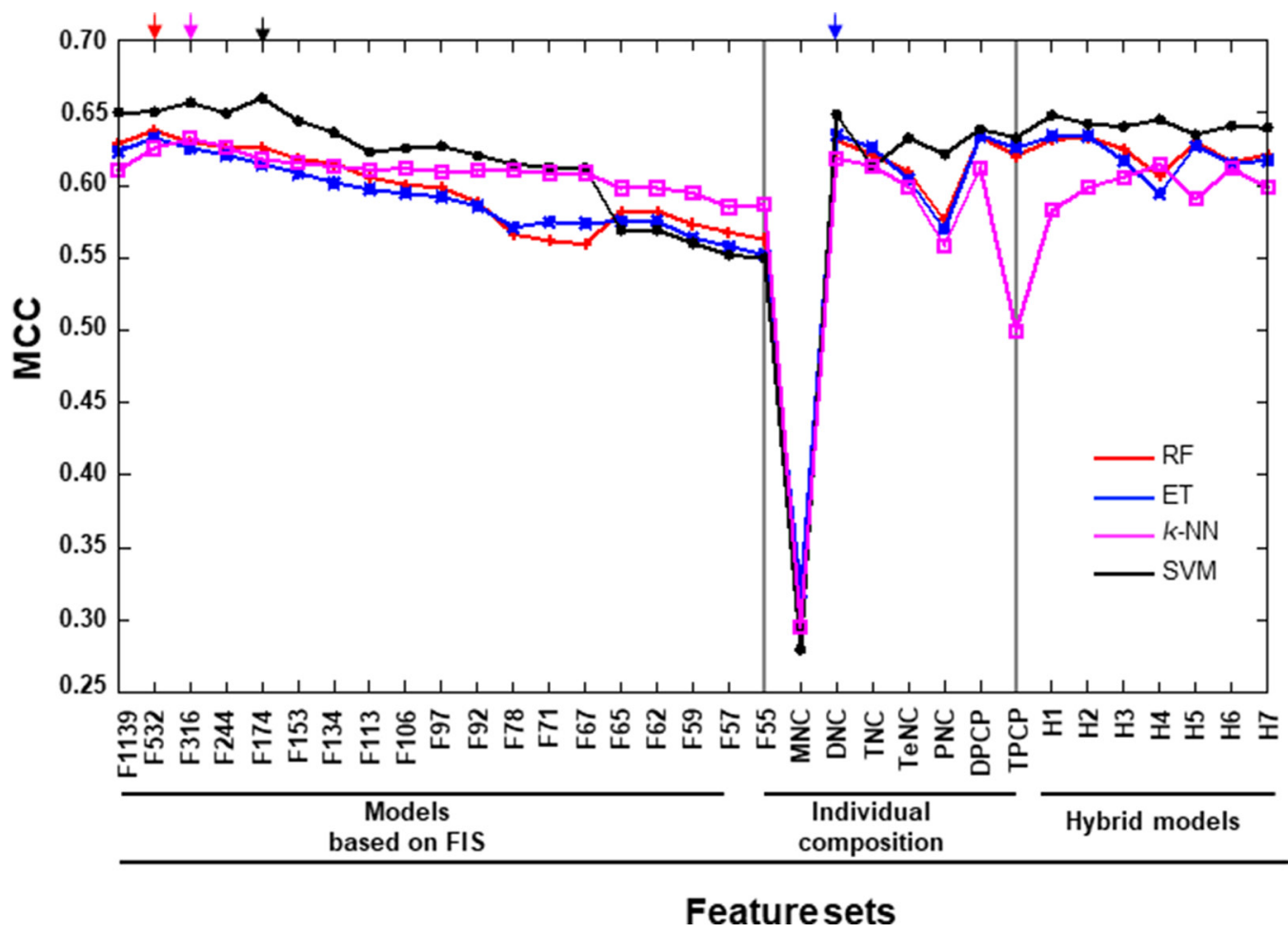

Figure 5: Performance of various prediction models on the benchmarking dataset. X-and Y-axes represent various featurebased prediction models and their performances measured in terms of MCC, respectively. The final selected prediction model for each MLbased method is indicated by arrows in different colors. Each point represents the average of five independent 10-fold cross-validations. The first 19 features are FX, where $\mathrm{X}$ is the number of features based on the FIS cut-off. H1: MNC+DNC; H2: H1+TNC; H3: H1+TeNC; H4: H3+PNC+DPCP; H5: DPCP+TPCP; H6: H1+TeNC+PNC+TPCP; and H7: H1+TNC+TeNC+PNC+H5. 
Table 2: Performance of the proposed DHSpred along with the state-of-the-art methods

\begin{tabular}{ccccccc}
\hline Method & MCC & Accuracy & Sensitivity & Specificity & Pt & Py \\
\hline DHSpred & $\mathbf{0 . 6 6 0}$ & $\mathbf{0 . 8 7 1}$ & $\mathbf{0 . 6 5 5}$ & $\mathbf{0 . 9 5 2}$ & $\mathbf{0 . 6 2 4}$ & $\mathbf{0 . 6 0 7}$ \\
iDHS-EL & 0.636 & 0.861 & 0.646 & 0.943 & 0.609 & 0.589 \\
RevcKmer & 0.616 & 0.852 & 0.654 & 0.928 & 0.607 & 0.582 \\
PseTNC & 0.610 & 0.861 & 0.607 & 0.946 & 0.574 & 0.553 \\
PseDNC & 0.571 & 0.837 & 0.611 & 0.923 & 0.563 & 0.533 \\
\hline
\end{tabular}

The first column represents the method name employed in this study. The second, the third, the fourth and the fifth, the sixth and the seventh respectively represent the MCC, accuracy, sensitivity, specificity, Pt and Py. Bold font denotes the best result.

great practical use for experimentalists. A few examples of bioinformatics tools/web server utilized for protein function predictions are shown in previous publications [27-33]. To this end, an online prediction server for DHSpred was developed, which is freely accessible at the following link: www.thegleelab.org/DHSpred.html. Users can paste or upload query DNA sequences in the FASTA format. After submitting the input DNA sequences, they can retrieve results in a separate interface. To maximize the convenience for users, step-by-step guidelines are provided in the Supplementary information. To ensure reproducibility of our findings, datasets used in this study can be downloaded from the DHSpred web server.

\section{DISCUSSION}

DHSs are nucleosome-free regions associated with different genomic regulatory elements, including promoters, enhancers, silencers, insulators, and transcription factor binding sites $[2-4,34]$. Therefore, identification of DHSs could be an effective approach for discovering functional DNA elements from noncoding sequences. Although many experimental methods have been proposed for identifying DHSs [7, 35], these methods are often expensive, laborious, and time-consuming for genome-wide application. Therefore, it is necessary to develop computational methods for predicting DHSs.

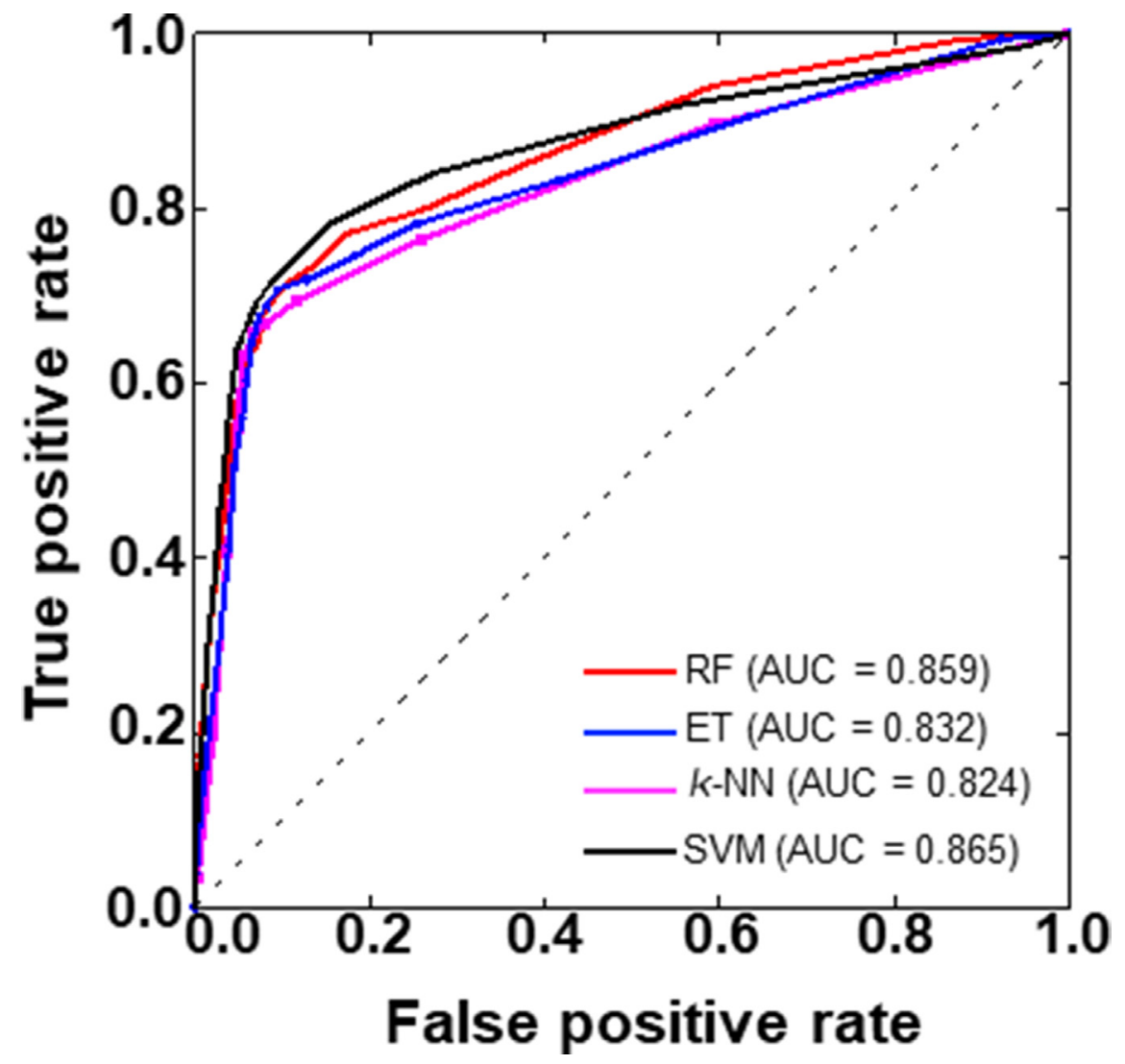

Figure 6: A graphical illustration to show the performances of four prediction models developed in this study via the ROC (receiver operating characteristics) curve. The area under the ROC curve is called AUC. Higher the AUC value is better the prediction performance. 
In this study, we developed a novel ML approach, called DHSpred, for predicting DHSs in the human genome. DHSpred combines various informative features from multiple sources, including $k$-mer, DPCP, and TPCP. Although $k$-mer and DPCP features have been used in earlier studies [9, 10, 12], this is the first report in which TPCP features were used. Interestingly, TPCP features contributed to $\sim 52 \%$ of the DHS prediction. In ML-based prediction methods, feature selection is an important step, as various data mining tasks have to deal with vast quantities of heterogeneous and possibly redundant features; feature selection has been widely used in various bioinformatics applications to improve the prediction performance [21,36-38]. Recently, a twostep feature selection protocol was applied in a protein model quality assessment method called SVMQA; the CASP assessors declared this method as the best one for selecting good-quality models from decoys [39]. We applied the same strategy for our current study and developed 132 prediction models using four ML-based methods with different sets of features. Among them, the SVM-based model using the optimal feature set showed the best performance.

In this study, it was demonstrated that DHSpred outperformed state-of-the-art methods (RevcKmer, PseDNC, PseTNC, and iDHS-EL) [9-12] and three other ML-based methods (RF, ET, and $k$-NN) by crossvalidations on the same benchmark dataset. The superior performance of our method might be attributed to two important factors: $(i)$ integration of previously reported features and inclusion of novel features that collectively make significant contributions to the performance; (ii) a two-step feature selection protocol to eliminate overlapping and redundant features. Furthermore, our approach is a general one, which is applicable to many other classification problems in structural bioinformatics. It can be readily extended for predicting DHSs in the plant genome.

The final identified optimal feature set through a rigorous feature selection protocol revealed that a major contribution from TPCP $(\sim 52 \%)$ and the remaining contribution (48\%) from DPCP, and important motifs in $k$-mers showed the best performance in distinguishing DHSs from non-DHSs, indicating a strong relationship between the optimal features and DHSs. Interestingly, all these features represent the local and global DNA sequence pattern, hence these properties might play a vital role in maintaining the DHS function. Although the current predictor is purely based on the primary sequences information, incorporating epigenetic information, including DNA methylation, and histone modifications will improve the performance of the predictor. Thus, we will also try to implement epigenetic information in our future work.

A user-friendly web interface is also available for researchers to use our prediction method. Indeed, our method is only the second method that is publicly available with high accuracy. Compared to experimental approaches, bioinformatics tools such as DHSpred represent a powerful and cost-effective approach for genome-wide prediction of DHSs. Therefore, DHSpred might be useful for large-scale DHS prediction and facilitate hypothesis-driven experimental design.

\section{MATERIALS AND METHODS}

According to the Chou's [40] five-steps guidelines that have been followed in a series of recent publications $[12,20,24-26,41-44]$, to develop a new prediction methods that can be easily used by both experimental scientists and also theoretical scientists, we should follow the following five guidelines: $(i)$ construct a valid benchmarking dataset to train and test the prediction model; (ii) formulate the biological sequence samples with an effective mathematical expression that can truly reflect their intrinsic correlation with the target to be predicted; (iii) introduce or develop a powerful algorithm (or engine) to operate the prediction; (iv) properly perform crossvalidation tests to objectively evaluate the anticipated accuracy of the predictor; $(v)$ establish a user-friendly web-server for the predictor that is accessible to the public. Below, we describe these steps one-by-one.

\section{Benchmarking dataset}

In this study, we utilized the dataset constructed by Noble et al. (2005), which was specifically used for studying DHSs [9]. We decided to use this dataset for a number of reasons. ( $i$ ) This dataset is more reliable, as it was constructed rigorously based on experimental data. (ii) It is a non-redundant dataset and none of the sequences has high pairwise sequence identity $(>80 \%)$ with any other sequence. Hence, this dataset could stringently exclude homology sequences. (iii) Most importantly, it will facilitate fair comparison between our method and the existing methods that were developed using the same benchmarking dataset.

Usually, the benchmark dataset comprises a training dataset and a testing dataset. The former is for training a model, while the latter is used for testing one. As pointed out in a comprehensive review [45], there is no need to artificially separate a benchmark dataset into training and testing datasets for validating a prediction method, as long as it is tested by the jackknife or subsampling (K-fold) cross-validation, as the outcome thus obtained is from a combination of different independent dataset tests. Thus, the benchmark dataset taken from Noble et al. [9] (2005) can be formulated as

$$
S=S^{+} \cup S^{-}
$$

where the positive subset $S^{+}$contained 280 DHS sequences, the negative subset $S^{\leftarrow}$ contained 737 nonDHS samples, and the symbol denotes union in the set 
theory. Thus, $S$ contained 1017 samples, which can be downloaded from our webserver.

\section{Feature extraction}

The aim of this experiment was to train each MLbased (SVM, RF, ETC, and $k$-NN) model to accurately map input features extracted from a nucleotide sequence to predict its class (i.e., DHS or non-DHS), which is considered a classification problem. We used nucleotide composition and di- and trinucleotide-physiochemical properties as input features. These features reflect the characteristics of a DNA sequence, from different perspectives, as defined below.

\section{$k$-mer composition}

MNC, DNC, TNC, TeNC, and PNC, whose feature vectors are essentially consisted of one dimensional arrays of size $4^{\mathrm{k}}$ and the $i^{\text {th }}$ element of the arrays is fraction of $k$-mer of type $i$.

$$
k-\text { mer }(i)=\frac{\text { Number of } k-\text { mer of type } i}{\text { Total number of } k-\text { mers of all types }}
$$

where $i=1,4^{k}$. Therefore, the size of feature vectors for MNC, DNC, TNC, TeNC, and PNC are 4, 16, 64, 256, and 1024 , respectively.

\section{DPCP}

In general, the spatial arrangement of two successive base pairs (bp) can be characterized by six quantities, which include three local translation parameters (shift, slide, and rise) and three local angular parameters (twist, tilt, and roll) [46, 47], which have been successfully used for many sequence-based classifications $[12,48]$. In this study, we also utilized DPCP for predicting DHSs. The parameter of six physicochemical properties is given in Supplementary Table 2, whose parameters were normalized in the range of $[0,1]$, based on the formula described in our previous studies [21, 49]. DPCP of a property can be calculated as follows:

$\operatorname{DPCP}$ of a property $(i)=\frac{\text { Total number of dipeptide }(\mathrm{j}) \times \operatorname{property}(i)}{\mathrm{N}_{\text {res }}}$

where $j$ is one of 16 possible dinucleotides, $\mathrm{N}_{\text {res }}$ is the sequence length, and $i$ is one of the six physicochemical properties.

\section{TPCP}

Recently, the twelve physiochemical properties (bendability [DNase], bendability [consensus], trinucleotide CG content; nucleosome positioning, consensus [roll], consensus [rigid], DNase I, DNase I [rigid], molecular weight [Daltons], molecular weight [kg], nucleosome, and nucleosome [rigid]) parameters had compiled for DNA trinucleotides [47]. The parameters for 12 physicochemical properties is given in the Supplementary Table 3, whose parameters were normalized in the range of $[0,1]$, based on the formula described in our previous studies [21, 49]. TPCP of a property can be calculated as follows:

TPCP of a property $(i)=\frac{\text { Total number of tripeptide }(\mathrm{j}) \times \operatorname{property}(i)}{\mathrm{N}_{\mathrm{res}}}$

where $j$ is one of 64 possible dinucleotides, $\mathrm{N}_{\text {res }}$ is the sequence length, and $i$ is one of the 12 physicochemical properties.

To the best of our knowledge, this is the first study in which $k$-mer, DPCP, and TPCP were considered for DHS prediction. Notably, TPCP has never been considered before, although $k$-mer, DNC, and DPCP are utilized in existing ML-based methods for DHS prediction $[9,10,12]$.

\section{Feature selection}

The feature selection protocol is the same as the one used in our recent study [21]. We used a two-step feature selection protocol to identify the most important features for predicting DHSs. In the first step, we applied the RF algorithm to estimate the importance of each feature. A detailed description of how we estimated the importance of input features has been published previously [21, 49]. Briefly, we used all the features as inputs for RF and carried out 10-fold cross-validation on the benchmark dataset. For each round of cross-validation, we built 10,000 trees, and the number of variables at each node was chosen randomly from between one to 100 . The ensemble average of FIS from all the trees is shown in Figure 3.

In the second step, we selected different sets of optimal feature candidates based on an FIS cut-off ( 0.0001 $\leq$ FIS $\leq 0.019$, with a step size of 0.0001$)$. The sets of features with FIS greater than the cut-off value were selected as the input features for the SVM classifier. For each feature set, we randomly divided our benchmark dataset into ten subsets ( $\sim 10 \%$ of DHSs and non-DHSs in each subset) for each validation step. At each crossvalidation step, nine subsets were then merged as the training dataset, in which $\mathrm{ML}$ parameters ( $C$ and $\mathrm{g}$ ) were optimized using a grid-search approach to train the model, while the remaining subsets were merged as the test set to validate the built model. This procedure was repeated ten times, and each subset was used in the training and validated in the testing. This 10 -fold crossvalidation procedure was repeated five times, resulting in five similar/different ML parameters and performances (see the section on Evaluation metrics). We considered the average performances and median ML parameters as the final stable values, and carried out performance evaluation. 


\section{Support vector machine}

SVM is a well-known supervised-ML technique used for developing both classification and regression models based on statistical theory [50]. A detailed description of an SVM has been reported previously $[21,51,52]$. A set of positive (DHS) and negative (non-DHS) samples were represented by the feature vectors with the corresponding labels. To classify the data as DHS or non-DHS, the SVM transforms input samples into one of two classes in a high-dimension feature space and learns an optimal decision boundary or hyperplane using kernel functions. Here, radial basis function (RBF) was set as the kernel function. An RBF-SVM requires the optimization of two critical parameters $(C$ : penalty constant and $\gamma$ : width). Hence, a grid search within the following ranges: $\mathrm{C}$ from $2^{-15}$ to $2^{10}$ and $\gamma$ from $2^{-10}$ to $2^{10}$ in $\log _{2}$ scale, was conducted to tune the SVM parameters ( $\mathrm{C}$ and $\gamma$ ). The optimal values for $C$ and $\gamma$ parameters for the final selected model were found to be 80.256 and 2.692, respectively. Besides SVM, we also utilized RF and other ML methods (ET and $k$-NN). These methods were implemented according to the scikit-learn package [53].

\section{Cross-validation}

In statistical prediction, the following three crossvalidation methods are often used to examine a predictor for its effectiveness in practical application: independent dataset test, subsampling test, and jackknife test. However, among these three methods, jackknife test is deemed as least arbitrary one which can always yield a unique result for a given benchmark dataset as elaborated in [54] and demonstrated by Eqs.28-30 in [40]. However, in order to reduce the computational time, we adopted 10 -fold crossvalidation as demonstrated by other investigators $[21,22$, 55-57].

\section{Evaluation metrics}

To compare the prediction methods, we evaluated sensitivity (Sn), specificity (Sp), accuracy (ACC), the Matthews correlation coefficient (MCC), product of Sn and $\mathrm{Sp}(\mathrm{Pt})$, and property excess (Py) $[12,58]$. Among these six metrics, Pt and Py helps in dealing with systems in which the number of negative samples is overwhelmingly greater than that of positive samples, as described by Jin et al. [58] (2005) and Yang et al. [59] (2005). The conventional formulae for these metrics lack intuitiveness and are not handy for most biologists, particularly MCC. Therefore, Chen et al. derived a new set of equations for these metrics [24, 25], based on Chou's symbols used in a study on protein signal peptide cleavage site [60]. The new formulae for these metrics are given in equation (5).

$$
\left\{\begin{array}{c}
\mathrm{Sn}=1-\frac{N_{-}^{+}}{N^{+}}, 0 \leq \mathrm{Sn} \leq 1 \\
\mathrm{Sp}=1-\frac{N_{+}^{-}}{N^{-}}, 0 \leq \mathrm{Sp} \leq 1 \\
\mathrm{ACC}=1-\frac{N_{-}^{+}+N_{+}^{-}}{N^{+}+N^{-}}, 0 \leq \mathrm{ACC} \leq 1 \\
\mathrm{MCC}=\frac{\left(\frac{N_{-}^{+}}{N^{+}}+\frac{N_{+}^{-}}{N^{-}}\right)}{\sqrt{\left(1+\frac{N_{-}^{+}-N_{+}^{-}}{N^{-}}\right)\left(1+\frac{N_{+}^{-}-N_{-}^{+}}{N^{+}}\right)}},-1 \leq \mathrm{MCC} \leq 1 \\
\mathrm{Pt}=\mathrm{Sn} \times \mathrm{Sp}, 0 \leq \mathrm{Pt} \leq 1 \\
\mathrm{Py}=1-(1-\mathrm{Sn})-(1-\mathrm{Sp}),-1 \leq \mathrm{Py} \leq 1
\end{array}\right.
$$

where $N^{+}$is the total number of the DHSs investigated, $N^{+}$is the number of DHSs incorrectly predicted as non-DHSs, $N^{-}$is the total number of nonDHSs investigated, and $N_{+}^{-}$is the number of non-DHSs incorrectly predicted as DHSs. The above set of metrics is valid only for the single-label systems. For the multi-label systems, whose existence has become more frequent in system biology [61] and system medicine [62-64], a completely different set of metrics is needed as defined in [65].

\section{Development of a prediction server}

An online prediction server was developed using hypertext markup language and Java script, with a Python script executing in the backend upon submission of DNA sequences in the FASTA format. Users can submit single or multiple sequences containing standard DNA bp in FASTA format. The DHSpred web server then outputs the results of SVM-based predictions, along with probability values.

\section{Statistical analysis}

The differences in MNC, DNC, TNC, TeNC, and PNC between ORIs and non-ORIs were analyzed using Welch's $t$-test. By providing the mean and standard deviation (SD) found, the data are presented as mean \pm standard error (SE). Also report the $p$-value and then draw a conclusion with $\alpha=0.01$, indicates that the difference is statistically meaningful. All statistical analysis was performed using our own script.

\section{Abbreviations}

DHSpred: DNase I hypersensitive site predictor; DNC: dinucleotide composition; DPCP: dinucleotide physicochemical properties; ET: extra tree classifier; $k$-NN: $k$-nearest neighbor: ML: machine learning; MNC: mononucleotide composition; PNC: penta nucleotide composition; RF: random forest; SVM: support vector machine (SVM); TeNC: tetra nucleotide composition; TNC: trinucleotide composition; TPCP: trinucleotide physicochemical properties. 


\section{Author contributions}

Conceived and designed the experiments: BM, GL. Performed the experiments: BM. Analyzed the data: BM, THS, GL. Contirbuted reagents/mateials/software tools: THS, GL. Wrote paper: BM, GL.

\section{ACKNOWLEDGMENTS AND FUNDING}

This work was supported by the Basic Science Research Program through the National Research Foundation (NRF) of Korea funded by the Ministry of Education, Science and Technology (2015R1D1A1A09060192), Priority Research Centers Program through the National Research Foundation of Korea (NRF) funded by the Ministry of Education, Science and Technology (2009-0093826), and the Brain Research Program through the National Research Foundation of Korea (NRF) funded by the Ministry of Science, ICT \& Future Planning (2016M3C7A1904392).

\section{CONFLICTS OF INTEREST}

The authors declare that they have no relevant conflicts of interest.

\section{REFERENCES}

1. Kodama Y, Nagaya S, Shinmyo A, Kato K. Mapping and characterization of DNase I hypersensitive sites in Arabidopsis chromatin. Plant Cell Physiol. 2007; 48:459-70.

2. Felsenfeld G. Chromatin as an essential part of the transcriptional mechanism. Nature. 1992; 355:219-24.

3. Felsenfeld G, Groudine M. Controlling the double helix. Nature. 2003; 421:448-53.

4. Gross DS, Garrard WT. Nuclease hypersensitive sites in chromatin. Annu Rev Biochem. 1988; 57:159-97.

5. Wu C, Bingham PM, Livak KJ, Holmgren R, Elgin SC. The chromatin structure of specific genes: I. Evidence for higher order domains of defined DNA sequence. Cell. 1979; 16:797-806.

6. Koohy H, Down TA, Hubbard TJ. Chromatin accessibility data sets show bias due to sequence specificity of the DNase I enzyme. PLoS One. 2013; 8:e69853.

7. Crawford GE, Holt IE, Whittle J, Webb BD, Tai D, Davis S, Margulies EH, Chen Y, Bernat JA, Ginsburg D, Zhou D, Luo S, Vasicek TJ, et al. Genome-wide mapping of DNase hypersensitive sites using massively parallel signature sequencing (MPSS). Genome Res. 2006; 16:123-31.

8. Song L, Crawford GE. DNase-seq: a high-resolution technique for mapping active gene regulatory elements across the genome from mammalian cells. Cold Spring Harb Protoc. 2010; 2010: pdb prot5384. https://doi.org/10.1101/ pdb.prot5384.
9. Noble WS, Kuehn S, Thurman R, Yu M, Stamatoyannopoulos J. Predicting the in vivo signature of human gene regulatory sequences. Bioinformatics. 2005; 21:i338-43.

10. Feng P, Jiang N, Liu N. Prediction of DNase I hypersensitive sites by using pseudo nucleotide compositions. Sci World J. 2014; 2014:740506.

11. Kabir M, Yu DJ. Predicting DNase I hypersensitive sites via un-biased pseudo trinucleotide composition. Chemom Intell Lab Syst. 2017; 167:78-84.

12. Liu B, Long R, Chou KC. iDHS-EL: identifying DNase I hypersensitive sites by fusing three different modes of pseudo nucleotide composition into an ensemble learning framework. Bioinformatics. 2016; 32:2411-18.

13. Di Filippo M, Bernardi G. Mapping DNase-I hypersensitive sites on human isochores. Gene. 2008; 419:62-65.

14. Federico C, Scavo C, Cantarella CD, Motta S, Saccone S, Bernardi G. Gene-rich and gene-poor chromosomal regions have different locations in the interphase nuclei of coldblooded vertebrates. Chromosoma. 2006; 115:123-28.

15. Saccone S, Federico C, Bernardi G. Localization of the gene-richest and the gene-poorest isochores in the interphase nuclei of mammals and birds. Gene. 2002; 300:169-78.

16. Cao R, Adhikari B, Bhattacharya D, Sun M, Hou J, Cheng J. QAcon: single model quality assessment using protein structural and contact information with machine learning techniques. Bioinformatics. 2017; 33:586-88.

17. Cao R, Bhattacharya D, Hou J, Cheng J. DeepQA: improving the estimation of single protein model quality with deep belief networks. BMC Bioinformatics. 2016; 17:495

18. Cao R, Cheng J. Protein single-model quality assessment by feature-based probability density functions. Sci Rep. 2016; 6:23990.

19. Cao R, Wang Z, Wang Y, Cheng J. SMOQ: a tool for predicting the absolute residue-specific quality of a single protein model with support vector machines. BMC Bioinformatics. 2014; 15:120.

20. Chen W, Feng P, Yang H, Ding H, Lin H, Chou KC. iRNAAI: identifying the adenosine to inosine editing sites in RNA sequences. Oncotarget. 2017; 8:4208-17. https://doi. org/10.18632/oncotarget.13758.

21. Manavalan B, Lee J. SVMQA: support-vector-machinebased protein single-model quality assessment. Bioinformatics. 2017; 33:2496-503.

22. Manavalan B, Basith S, Shin TH, Choi S, Kim MO, Lee G. MLACP: machine-learning-based prediction of anticancer peptides. Oncotarget. 2017; 8:77121-36. https://doi. org/10.18632/oncotarget.20365.

23. Tang H, Cao RZ, Wang W, Liu TS, Wang LM, He CM. A two-step discriminated method to identify thermophilic proteins. Int J Biomath. 2017; 10:1750050. 
24. Chen W, Feng PM, Lin H, Chou KC. iRSpot-PseDNC: identify recombination spots with pseudo dinucleotide composition. Nucleic Acids Res. 2013; 41:e68.

25. Chen W, Tang H, Ye J, Lin H, Chou KC. iRNA-PseU: identifying RNA pseudouridine sites. Mol Ther Nucleic Acids. 2016; 5:e332.

26. Liu B, Yang F, Chou KC. 2L-piRNA: A Two-Layer Ensemble Classifier for Identifying Piwi-Interacting RNAs and Their Function. Mol Ther Nucleic Acids. 2017; 7:26777.

27. Basith S, Manavalan B, Gosu V, Choi S. Evolutionary, structural and functional interplay of the I $\mathrm{B}$ family members. PLoS One. 2013; 8:e54178.

28. Basith S, Manavalan B, Govindaraj RG, Choi S. In silico approach to inhibition of signaling pathways of Toll-like receptors 2 and 4 by ST2L. PLoS One. 2011; 6:e23989.

29. Govindaraj RG, Manavalan B, Basith S, Choi S. Comparative analysis of species-specific ligand recognition in Toll-like receptor 8 signaling: a hypothesis. PLoS One. 2011; 6:e25118.

30. Govindaraj RG, Manavalan B, Lee G, Choi S. Molecular modeling-based evaluation of hTLR10 and identification of potential ligands in Toll-like receptor signaling. PLoS One. 2010; 5:e12713.

31. Manavalan B, Basith S, Choi YM, Lee G, Choi S. Structurefunction relationship of cytoplasmic and nuclear $\mathrm{I} \kappa \mathrm{B}$ proteins: an in silico analysis. PLoS One. 2010; 5:e15782.

32. Manavalan B, Govindaraj R, Lee G, Choi S. Molecular modeling-based evaluation of dual function of $\mathrm{I} \kappa \mathrm{B} \zeta$ ankyrin repeat domain in toll-like receptor signaling. J Mol Recognit. 2011; 24:597-607.

33. Manavalan B, Murugapiran SK, Lee G, Choi S. Molecular modeling of the reductase domain to elucidate the reaction mechanism of reduction of peptidyl thioester into its corresponding alcohol in non-ribosomal peptide synthetases. BMC Struct Biol. 2010; 10:1.

34. Thurman RE, Rynes E, Humbert R, Vierstra J, Maurano MT, Haugen E, Sheffield NC, Stergachis AB, Wang H, Vernot B, Garg K, John S, Sandstrom R, et al. The accessible chromatin landscape of the human genome. Nature. 2012; 489:75-82.

35. Dorschner MO, Hawrylycz M, Humbert R, Wallace JC, Shafer A, Kawamoto J, Mack J, Hall R, Goldy J, Sabo PJ, Kohli A, Li Q, McArthur M, Stamatoyannopoulos JA. Highthroughput localization of functional elements by quantitative chromatin profiling. Nat Methods. 2004; 1:219-25.

36. Li F, Li C, Wang M, Webb GI, Zhang Y, Whisstock JC, Song J. GlycoMine: a machine learning-based approach for predicting $\mathrm{N}$-, C- and O-linked glycosylation in the human proteome. Bioinformatics. 2015; 31:1411-19.

37. Wang M, Zhao XM, Takemoto K, Xu H, Li Y, Akutsu T, Song J. FunSAV: predicting the functional effect of single amino acid variants using a two-stage random forest model. PLoS One. 2012; 7:e43847.
38. Zheng C, Wang M, Takemoto K, Akutsu T, Zhang Z, Song $\mathrm{J}$. An integrative computational framework based on a twostep random forest algorithm improves prediction of zincbinding sites in proteins. PLoS One. 2012; 7:e49716.

39. Kryshtafovych A, Monastyrskyy B, Fidelis K, Schwede T, Tramontano A. Assessment of model accuracy estimations in CASP12. Proteins. 2017 Aug 17. [Epub ahead of print].

40. Chou KC. Some remarks on protein attribute prediction and pseudo amino acid composition. J Theor Biol. 2011; 273:236-47.

41. Cheng X, Zhao SG, Xiao X, Chou KC. iATC-mISF: a multi-label classifier for predicting the classes of anatomical therapeutic chemicals. Bioinformatics. 2017; 33:2610.

42. Feng P, Ding H, Yang H, Chen W, Lin H, Chou KC. iRNAPseColl: Identifying the Occurrence Sites of Different RNA Modifications by Incorporating Collective Effects of Nucleotides into PseKNC. Mol Ther Nucleic Acids. 2017; 7:155-63.

43. Khan M, Hayat M, Khan SA, Iqbal N. Unb-DPC: identify mycobacterial membrane protein types by incorporating unbiased dipeptide composition into Chou's general PseAAC. J Theor Biol. 2017; 415:13-19.

44. Meher PK, Sahu TK, Saini V, Rao AR. Predicting antimicrobial peptides with improved accuracy by incorporating the compositional, physico-chemical and structural features into Chou's general PseAAC. Sci Rep. 2017; 7:42362.

45. Chou KC. Structural bioinformatics and its impact to biomedical science. Curr Med Chem. 2004; 11:2105-34.

46. Chen W, Lin H, Chou KC. Pseudo nucleotide composition or PseKNC: an effective formulation for analyzing genomic sequences. Mol Biosyst. 2015; 11:2620-34.

47. Liu B, Liu F, Fang L, Wang X, Chou KC. repDNA: a Python package to generate various modes of feature vectors for DNA sequences by incorporating user-defined physicochemical properties and sequence-order effects. Bioinformatics. 2015; 31:1307-09.

48. Lin H, Deng EZ, Ding H, Chen W, Chou KC. iPro54PseKNC: a sequence-based predictor for identifying sigma-54 promoters in prokaryote with pseudo k-tuple nucleotide composition. Nucleic Acids Res. 2014; 42:12961-72.

49. Manavalan B, Lee J, Lee J. Random forest-based protein model quality assessment (RFMQA) using structural features and potential energy terms. PLoS One. 2014; 9:e106542.

50. Vapnik VN. An overview of statistical learning theory. IEEE Trans Neural Netw. 1999; 10:988-99.

51. Manavalan B, Kuwajima K, Joung I, Lee J. (2015). Structure-based protein folding type classification and folding rate prediction. Bioinformatics and Biomedicine (BIBM), 2015 IEEE International Conference on: IEEE), pp. 1759-1761. https://doi.org/10.1109/ BIBM.2015.7359953. 
52. Uziela K, Shu N, Wallner B, Elofsson A. ProQ3: improved model quality assessments using Rosetta energy terms. Sci Rep. 2016; 6:33509.

53. Abraham A, Pedregosa F, Eickenberg M, Gervais P, Mueller A, Kossaifi J, Gramfort A, Thirion B, Varoquaux G. Machine learning for neuroimaging with scikit-learn. Front Neuroinform. 2014; 8:14.

54. Chou KC, Shen HB. Cell-PLoc: a package of Web servers for predicting subcellular localization of proteins in various organisms. Nat Protoc. 2008; 3:153-62.

55. Ding H, Feng PM, Chen W, Lin H. Identification of bacteriophage virion proteins by the ANOVA feature selection and analysis. Mol Biosyst. 2014; 10:2229-35.

56. Gupta S, Sharma AK, Shastri V, Madhu MK, Sharma VK. Prediction of anti-inflammatory proteins/peptides: an insilico approach. J Transl Med. 2017; 15:7.

57. Sharma AK, Jaiswal SK, Chaudhary N, Sharma VK. A novel approach for the prediction of species-specific biotransformation of xenobiotic/drug molecules by the human gut microbiota. Sci Rep. 2017; 7:9751.

58. Jin Y, Dunbrack RL Jr. Assessment of disorder predictions in CASP6. Proteins. 2005; 61:167-75.
59. Yang ZR, Thomson R, McNeil P, Esnouf RM. RONN: the bio-basis function neural network technique applied to the detection of natively disordered regions in proteins. Bioinformatics. 2005; 21:3369-76.

60. Chou KC. Prediction of protein signal sequences. Curr Protein Pept Sci. 2002; 3:615-22.

61. Chou KC, Wu ZC, Xiao X. iLoc-Hum: using the accumulation-label scale to predict subcellular locations of human proteins with both single and multiple sites. Mol Biosyst. 2012; 8:629-41.

62. Cheng X, Zhao SG, Xiao X, Chou KC. iATC-mISF: a multi-label classifier for predicting the classes of anatomical therapeutic chemicals. Bioinformatics. 2017; 33:341-46.

63. Cheng X, Zhao SG, Xiao X, Chou KC. iATC-mHyb: a hybrid multi-label classifier for predicting the classification of anatomical therapeutic chemicals. Oncotarget. 2017; 8:58494-503. https://doi.org/10.18632/oncotarget.17028.

64. Qiu WR, Sun BQ, Xiao X, Xu ZC, Chou KC. iPTM-mLys: identifying multiple lysine PTM sites and their different types. Bioinformatics. 2016; 32:3116-23.

65. Chou KC. Some remarks on predicting multi-label attributes in molecular biosystems. Mol Biosyst. 2013; 9:1092-100. 